\section{FROM SECURE ENVIRONMENT TO SECURE EXPERIENCE}

Leonardo Baumworcel. Hospital São Lucas- Sergipe

\subsection{6/leader-2019-FMLM.85}

Today all hospitals have to add some new skills related to patient earned value. The challenge is to convert all stakeholders the patient earned value. Since January 2019, we measure the net promoter score of our client. Every client of the hospital receives at home the net promoter score questionaries. Our task is to generate information about this questionaries daily. To achieve that goal, we schedule meetings first to make a diagnosis of how we are going to modify the crew behavior related to the patient experience. To disseminate this point of view we send by the social network every day the net promoter score measurement of each hospital unit and some testimonials. The interventions done were meetings with the crew to the goal to translate the vocabulary and the culture of safety to safety and rapport. In this meeting with all the hospital crew, we learned the points to change the personal behavior that was needed and the institutional process. The board of the hospital has the task to facilitate all this action. The first month of this project, we made a diagnosis. In the second month, we begin to implement these meeting and this massive dissemination of the lessons learned to everybody. During this process, the director made a one-to-one meeting with some critical stakeholders to keep them on track. The net promoter score is growing each month, and now, we have some areas of excellence. The direct comparison of each net promoter score of our holding is distributed daily for the hospital directors. The principal challenge was how to change the actual culture that it was working well since the beginning of the accreditation setting toward a better-experienced level with safety. The lesson learned is to focus on patient experience since entry through the hospital.

\section{PATIENT EXPERIENCE ON THE CORRIDOR: ARE PEOPLE ANXIOUS? IF SO, WHAT CAN WE DO ABOUT IT?}

Timothy Mossad. Consultant in Emergency Medicine, UK

10.1136/leader-2019-FMLM.86

Attendances to Emergency Departments (EDs) have grown over recent years. Many patients are experiencing delays in moving from ED to their acute admission destination.

This quality improvement project explored qualitative aspects to perception of corridor care. It provides a framework for ongoing learning, continuing to put patients at the focus of care, and facilitates the development of good practice for those journeying through busy EDs.

I aimed to reduce levels of state anxiety experienced by patients in our ED corridor, by one metric point as measured across a Likert scale of agreement.

Following trust and governance approval, SWOT (strengths, weaknesses, opportunities, threats) and stakeholder analyses, 185 service users participated in standardised questionnaire completion over five cycles of a PDSA (plan, do, study, act) model over eight months. Demographic data was collected.

Interventions included; apology and space allocation, named nurse, healthcare assistant round, pain scoring and patient enquiry, curtains for privacy and dignity.
Sequenced interventions mapped patient anxiety measurements pre and post intervention using the well-validated Spielberg state anxiety tool. A Likert scale of agreement (metrically converted: strongly disagree -2 to strongly agree +2 ) measured patients' perception of care throughout, based on common themes identified from SWOT analysis.

Overall, metric levels of 'bad' anxiety feelings [tense/worried/upset] reduced and 'good' anxiety feelings [calm/content/ relaxed] increased with progressive intervention sequencing, $\mathrm{p}=0.0195$ (0.000016-0.09928, variance 0.5, t-test).

Although people recognised attempts to manage their anxiety, these attempts did not have significant effects on their overall level of anxiety; $\mathrm{p}=0.271(0.055-0.496)$ for 'good' anxiety and $p=0.25(0.067-0.402)$ for 'bad' anxiety.

Further work is required with larger studies and allowing for multivariate analysis to explore this in the wider ED setting.

\section{LEADERSHIP AND MANAGEMENT SKILLS FOR NEW CLINICAL ONCOLOGY TRAINEES - HAS A PRESCRIPTIVE APPROACH RESULTED IN POOR COMPLIANCE?}

${ }^{1}$ Thomas Charlton, ${ }^{2}$ Anita Cheah, ${ }^{2}$ Priya Thakrar, ${ }^{2}$ Emma Bailey, ${ }^{2}$ Alex Thomas, ${ }^{2}$ Sian Kitchen, ${ }^{2}$ Jo Szram. ${ }^{1}$ Imperial College Healthcare NHS Trust; ${ }^{2}$ Health Education UK

\subsection{6/leader-2019-FMLM.87}

Background Advances in cancer treatment continue at rapid pace, patient numbers are increasing and there is a significant workforce shortage. Strong leadership and management is required.

Leadership and management features heavily in the RCR's 2016 Clinical Oncology (CO) Syllabus, however, there is limited scope for formal development in this area.

The Spiral Leadership Toolkit (SLT) was introduced to help trainees practically develop these competencies during training. Based on the NHS Leadership Academy's Healthcare Leadership Model, it includes eight domains, e.g. finance and risk.

Intervention A pilot study involving all new South London CO ST3 trainees (September 2019). The objective was to complete at least two projects from the eight domains during ST3.

To demonstrate compliance, trainees were advised to upload evidence onto their e-portfolios as part of ARCP (May 2019).

A midpoint, cross-sectional survey was distributed to trainees after 6 months to understand how the SLT had been used and any challenges faced.

Results 14/15 (93\%) trainees responded. Only 4/14 (29\%) had used the SLT to complement their training. 7/14 (50\%) trainees had included SLT in their Personal Development Plan (PDP).

In terms of barriers, $9 / 12(75 \%)$ felt that they had received neutral or limited support from their trust. $7 / 14$ (50\%) stated that they didn't have time to participate.

Written comments included 'Another hoop to jump through' and a 'mandatory form filling exercise'.

Conclusion There has been a disappointing uptake with only a third of trainees using the SLT. Two major barriers include a lack of time and support from trusts. Half of trainees have included the SLT in their PDP, demonstrating a future intention to participate. 
It is clear that trainees are struggling to balance multiple commitments and that the SLT is being viewed by some as another 'tick box' exercise. Hopefully some trainees have found it a useful tool for developing vital skills.

\section{IMPROVING PATIENTSAFETY CULTURE IN THE PRIMARY CARE SETTING}

${ }^{1}$ A Hollis*, 'Leo Feinberg, ${ }^{2}$ Will Murdoch. 'Sandwell and West Birmingham NHS Trust, Birmingham, West Midlands; ${ }^{2}$ The Modality Partnership, Birmingham, West Midlands

10.1136/leader-2019-FMLM.88

Aims To improve engagement with the incident reporting process and to encourage staff to raise issues and create a proactive culture of quality improvement.

Methods All staff members were invited to take part in the GP Safety Climate Questionnaire pre and post intervention to assess self-reported feelings towards patient safety at the practice. We then developed a novel incident reporting tool which was introduced in collaboration with an adapted 'Take off, cruising and landing' daily safety huddle. The incident reporting tool was designed to be as simple as possible, easy to access and quick to complete.

Results Prior to the new tool and adapted safety huddle we had 6 significant event forms completed within the practice in the preceding three months. Following implementation, we had a total of 191 incident forms over a ten-week period (which aimed to capture all issues from low level to significant events). These issues highlighted several process issues within our GP practice namely issues with appointments, inter-practice communication and prescriptions. The safety huddle also allowed issues to handed over from one day to the next formally. GP safety climate questionnaire scores also showed a substantial improvement with below average scores (compared with other practices) pre implementation improving to above average scores post.

Conclusions This project demonstrates that a relatively simple intervention can have effect significant positive cultural change in an organisation over a small period of time. Through this project we were able to generate a wealth of information that we could use to target areas of improvement. By giving frontline staff a mechanism to record issues it is possible to develop a positive culture of grass roots change. Incident reporting can act as a vehicle not only to improve patient safety but more broadly to generate ongoing ideas for quality improvement within an organisation.

\section{IMPROVING CARDIOLOGY REFERRAL PROCESS AT ROYAL SURREY COUNTY HOSPITAL}

P Bamania*, A Mellan, K Moosai, Z Zuberi. Royal Surrey County Hospital, Guildford, UK

\subsection{6/leader-2019-FMLM.89}

Background The Cardiology referral system at Royal Surrey County Hospital involved bleeping the registrar. It is often the junior doctors' job to make the referral, and occasionally not all information is at hand.

Aim To improve efficiency of inpatient Cardiology referral process by creating an easy-to-use system enabling effective timely referrals with relevant information.
Methods Questionnaires were distributed to junior doctors with five closed questions, rated strongly agree to strongly disagree. A proforma was designed and implemented with feedback from the Cardiology team. Improvements were measured objectively through questionnaires and verbal feedback.

Leadership This project began with identifying an area for improvement, reviewing and planning for standardised system. It involved working closely with the Cardiology team, through meetings and email correspondence. We also took into consideration suggestions from clinicians outside Cardiology. We evaluated referral systems in other specialities, which helped aid design.

Results Before implementation: 22 junior doctors responded. $50 \%$ agreed they knew how make to make a Cardiology referral, $50 \%$ disagreed. $68 \%$ disagreed that it is very easy to get a referral. 50\% agreed they included the correct information. $55 \%$ agreed they were often told to gather more information. $77 \%$, spent two hours or more trying to refer.

Two months after implementation: 21 junior doctors responded. $81 \%$ agreed they knew how make to make a Cardiology referral. $67 \%$ agreed that it is very easy to get a cardiology referral. 57\% agreed they always included the correct information. 24\% agreed they were asked to gather more information. All referrals were completed within an hour.

Conclusion The implementation of these changes highlights the key qualities needed in leadership and management good communication skills, being innovative as well as challenges one may face and being able to use feedback to guide positive change.

\section{YOUR'RE HIRED! DEVELOPING CLINICAL LEADERS OF TOMORROW USING THE GOVERNMENT APPRENTICESHIP LEVY}

${ }^{1}$ Bill Kawai-Calderhead, ${ }^{2}$ Roddy Christie. ${ }^{1}$ Royal Army Medical Corps, British Army; ${ }^{2}$ Always Consult Ltd, UK

\subsection{6/leader-2019-FMLM.90}

Aim Medical leadership is recognised as an essential facet of clinical practice. However there lacks standardised, sustainable training for postgraduate doctors in particular to support the transition from undergraduate medical student to Foundation Year 1 (FY1) doctor. This challenging transition creates significant anxiety amongst FY1s and clinician burnout is a national concern. Through the Government Apprenticeship Scheme NHS Trusts have access to a ring-fenced budget that can provide funded, vocationally based, nationally benchmarked leadership and management (LM) training which can support FY1s during this transition.

Methods In December 2017, through a partnership between the authors, South Tees Hospitals NHS Foundation Trust and Always Consult, a Registered Apprenticeship Training provider, the Foundation Leadership and Management (FLM) programme for FY1s was launched. FLM consists of 12 LMthemed modules which complement the FY1 clinical curriculum and leads to a nationally recognised qualification enabling membership of two leading international LM bodies. Participants are regularly surveyed for their self-rated preparedness for the LM challenges of FY1 practice and resilience through the Brief Resilience Score (BRS). 